\title{
Overexpression of miR-126 Inhibits the Activation and Migration of HSCs through Targeting CRK
}

\author{
Xu-Hua Gonga,f Chao Chen bef Peng Hou b,f Song-Cheng Zhu ${ }^{c}$ Chao-Qun Wu ${ }^{d}$ \\ Chen-Lin Song ${ }^{c} \mathrm{Wu} \mathrm{Ni}^{\mathrm{e}}$ Jiang-Feng Hua Ding-Kang Yao ${ }^{a}$ Jiu-Hong Kang ${ }^{c}$ \\ Liang Zhu ${ }^{\mathrm{a}}$ \\ aDepartment of Gastroenterology, Changzheng Hospital, Second Military Medical University, Shanghai, \\ bDepartment of Gastroenterology, The First Affiliated Hospital of Chinese PLA General Hospital, Beijing, \\ 'Clinical and Translational Research Center of Shanghai First Maternity \& Infant Health Hospital, \\ Shanghai Key Laboratory of Signaling and Disease Research, School of Life Science and Technology, \\ Tongji University, Shanghai, ' ${ }^{\circ}$ enetics Institute, Fudan University, Shanghai, e Department of Infectious \\ Diseases, Changzheng Hospital, Second Military Medical University, Shanghai, China; ${ }^{\text {TThese authors }}$ \\ contributed equally to this work
}

\section{Key Words}

miR-126 • HSCs • Activation • CRK

\begin{abstract}
Background \& Aims: MicroRNAs (miRNAs) have been shown to play essential roles in HSCs activation which contributes to hepatic fibrosis. Our previous miRNA microarray results suggested that miR-126 might be decreased during HSCs activation as other studies. The aim of this study is to investigate the role of miR-126 during HSCs activation. Methods: In this study, the expression of miR-126 during HSCs activation was measured and confirmed by qRT-PCR. Then, miR-126 expression was restored by transfection of lentivirus vector encoding miR-126. Futhermore, cell proliferation was assayed by the cell counting kit-8 (CCK-8), cell migration was assayed by transwell assay, and the markers of activation of HSCs, $\alpha-S M A$ and collagen type I, were assayed by qRT-PCR, Western Blotting, Immunostaining and ELISA. Luciferase reporter assay was used to find the target of miR-126, and Western Blotting and Immunostaining was used to validate the target of miR-126. Then, the expression and the role of the target of miR-126 during HSCs activation was further assessed. Results: The expression of miR-126 was confirmed to be significantly decreased during HSCs activation. Overexpression of miR-126 significantly inhibited HSCs migration but did not affect HSCs proliferation. The expression of $\alpha-S M A$ and collagen type I were both obviously decreased by miR-126 restoration. CRK was found to be the target of miR-126 and overexpression of miR-126 significantly inhibited CRK expression. And it was found that overexpression of CRK also significantly decreased miR-126 expression and promoted HSCs activation. Conclusions: Our study showed that overexpression of miR-126 significantly inhibited the activation and migration of HSCs through targeting CRK which can also decrease miR-126 expression and promote HSCs activation.
\end{abstract}

Copyright (C) 2014 S. Karger AG, Basel 


\section{Cellular Physiology and Biochemistry}

Cell Physiol Biochem 2014;33:97-106

DOI: 10.1159/000356653

onine: January 17, 2014

\section{Introduction}

Hepatic fibrosis is characterized by excessive deposition of extracellular matrix (ECM) components, predominantly type I collagen [1]. HSCs are the principal cell type promoting synthesis and deposition of ECM. After hepatic injury, HSCs transdifferentiate from quiescent phenotype into activated phenotype, migrate to the injured region and produce ECM which contribute greatly to the progression of hepatic fibrosis [2]. miRNAs are small, endogenous, non-coding RNAs that interact with the untranslated region (3'UTR) of the target mRNAs and result in inhibition of translation or degradation of mRNA. miRNAs have been found to play an important role in regulating cell proliferation, migration, apoptosis and differentiation, thus are involved in the development of many diseases. At present many miRNAs has also been validated to play essential roles in HSCs activation and hepatic fibrosis. miR-15b and miR-16 inhibited Bcl-2 and caspase signaling pathway and thus induced HSCs apoptosis [3]. Down-regulation of miR-27a and 27b through targeting retinoid X receptor $\alpha \mathrm{RXR} \alpha$ allowed activated HSCs to switch to quiescent phenotype, with restored cytoplasmic lipid droplets and decreased cell proliferation [4]. Overexpression of miRNA-150 and miRNA-194 inhibited HSCs activation through targeting c-myb and rac1 respectively [5]. Overexpression of miR29 suppressed HSCs activation, proliferation and ECM production through directly targeting collage type I and other target gene such as SP1 and c-fos and so on [6]. miR-221/222 were increased during HSCs activation and the progression of liver fibrosis [7]. miR-34 family was upregulated and targeted ACSL1 in dimethylnitrosamine-induced hepatic fibrosis in rats [8]. miR-21 enhanced TGF- $\beta$ signaling pathway and promoted hepatic fibrogenesis by targeting SMAD7 [9]. miR-195 inhibited cyclin E1 expression and contributed to interferon- $\beta$-induced inhibition of HSCs proliferation [10].

Our previous miRNA microarray results showed that miR-126 was significantly decreased during HSCs activation [11], which was consistent with others'researches. However this has not yet been confirmed by qRT-PCR and the role of miR-126 during HSCs activation has not been studied at present. Previous researches suggested that miR-126 was important in regulating cell proliferation, apoptosis, development and differentiation in many cell types, thus involved in the development of many diseases, especially in cancers. miR-126 inhibited lung cancer cells proliferation by targeting EGFL7 and VEGF in vitro and in vivo [12, 13]. In human gastric cancer miR-126 also functioned as a tumour suppressor [14]. miR126 suppressed the growth of neoplastic cells by targeting phosphatidylinositol 3-kinase signaling and was frequently decreased in colon cancers [15]. As miR-126 often presented to be a growth and differentiation suppressor in many cancer cells and its expression may be significantly decreased during HSCs activation so we hypothesize that miR-126 may also played essential roles in HSCs activation and differentiation.

In this study, miR-126 was confirmed to be significantly decreased during HSCs activation by qRT-PCR corresponding to our previous miRNA microarray results showed. Overexpression of miR-126 in HSCs inhibited HSCs migration while did not affect HSCs proliferation. And $\alpha$-SMA and collagen type I were also both obviously decreased by miR-126 restoration. CRK, which played an integral role in determining cell shape and locomotion, was found to be a target of miR-126. Overexpression of miR-126 decreased CRK expression which was found to be able to promote HSCs activation. These results demonstrated that overexpression of miR-126 inhibited HSCs activation may via inhibiting CRK, which could decrease miR-126 expression and promote HSCs activation vice versa. miR-126/CRK pathway is a novel mechanism in regulating HSCs activation and migration, and may be a potential therapeutic target for hepatic fibrosis in future.

\section{Materials and Methods}

Isolation and culture of rat HSCS

Normal male Sprague-Dawley rats, weighing about 400-500 g each, were cared for according to the principles of guide for the care and use of laboratory animals formulated by the Second Military Medical 


\section{Cellular Physiology and Biochemistry}

Cell Physiol Biochem 2014;33:97-106

DOI: $10.1159 / 000356653$

Published online: January 17, 2014

Gong et al.: miR-126 Inhibits HSCs Activation
(C) 2014 S. Karger AG, Basel

www.karger.com/cpb

Table 1. Primers used in qRT-PCR

\begin{tabular}{lllc}
\hline Gene & Forward primer $\left(5^{\prime}-3^{\prime}\right)$ & Reverse primer $\left(5^{\prime}-3^{\prime}\right)$ & Productsize $(\mathrm{bp})$ \\
\hline$a-S M A$ & CCGAGATCTCACCGACTACC & TCCAGAGCGACATAGCACAG & 120 \\
Col I & AGCTGCATACACAATGGCCTAA & CCTATGACTTCTGCGTCTGGTG & 209 \\
Actin & ACCGTGAAAAGATGACCCAGAT & AACCCTCATAGATGGGCACAGT & 162
\end{tabular}

University. Primary rat HSC were isolated from the livers through two steps of digestion as our previously research did [11].

The isolated HSCs and HSC-T6 cell line were cultured in High-glucose DMEM (Hyclone), supplemented with10\% FBS (BIOCHROM AG), plus 1×penicillin/streptomycin (P/S) (Thermo) and 2 mmol/l glutamine. Primary rat HSC were harvested at day 2 , day 7 and day 14 , respectively.

Growth curve

Three days after infection with lenti-virus, cells were transferred into 96-well plates at a density of 2000 cells per well cultured for 24, 48, 72 and 96 hours. Cell growth was tested in triplicate using Cell Count Kit-8 (CCK-8) (Dojindo, Kumamoto, Japan) according to the manufacturer's protocol.

Lentiviral construction and infection.

Lentivector Expression System was purchased from Shanghai SunBio Medical Biotechnology Co., Ltd. Packaging and production of lentivirus were performed according to the manufacturer's protocol. The recombinant miRNA expression vectors (MS1022X-miR-126) and Lentivirus Package plasmid mix were co-transfected into 293TN cells with Lipofectamine 2000 (Invitrogen). HSC-T6 were cultured in 6-well uncoated plastic plates at a density of $1 \times 10^{5} / \mathrm{ml}$ and divided into two groups: miR-126 overexpression group (MS1022X-miR-126) and negative control group (pGIPZ). HSC-T6 was infected with MOI 30 for $12 \mathrm{~h}$ at $37^{\circ} \mathrm{C}$, followed by addition of fresh growth medium. Three days later, cells infected with lentiviral vectors expressed enhanced green fluorescent protein (GFP). Cells were harvested three days after infection for RNA, miRNA and protein isolation.

\section{Real-Time PCR}

Total RNA was extracted from cells using the TRNzol-A+ total RNA Extract Kit (TIANGEN) according to the manufacturer's instructions. cDNA synthesis was performed with the TIANScript RT Kit (TIANGEN), following the manufacturer's instructions with a reaction volume of $20 \mu \mathrm{l}$. For real-time amplification, the template was equivalent to $20 \mathrm{ng}$ of total RNA. 40 cycles of quantitative PCR was normalized to the endogenous $\beta$-actin gene. Primer sequences for endogenous $\beta$-actin, collagen type I, $\alpha$-SMA and CRK were listed in Table 1. For miRNA quantitative analysis, total RNA was extracted using the method above. Subsequently, we used the Bulge-LoopTM miRNA qPCR Primer Set (Ruibobio Co. Cat\#MQP-0101) to detect the expression of miRNAs by qRT-PCR assay with the SYBR Green qPCR Master Mix (Tiangen). The relative quantitative value was expressed by the 2- $\Delta$ Ct method (Livak KJ, et al. 2001), representing the amount of candidate miRNA expression with the same calibrators and U6 as the internal normalized control. Each experiment was performed in duplicates and repeated three times.

\section{Western Blotting}

Cells were lysed in $1 \times$ lysis buffer that was diluted from $5 \times$ lysis buffer $(0.5 \mathrm{~mol} / \mathrm{L} \mathrm{Tris} \cdot \mathrm{HCl}(\mathrm{pH} 6.8)$ $2.5 \mathrm{ml}$ DTT $0.39 \mathrm{~g}$, SDS $0.5 \mathrm{~g}$, bromophenol blue $0.025 \mathrm{~g}$, glycerine $2.5 \mathrm{ml}$ ). Equal amount of the cell lysis was separated on polyacrylamide-SDS gels, blotted on nitrocellulose filter membrane and probed with the following primary antibodies: anti-collagen type I (Abcam, 1:1000), anti- $\alpha$-SMA (sigma, 1:1000), anti-CRK (Epidomics, 1:1000), anti- $\alpha$-tubulin (sigma, 1:2500), anti-GAPDH (Sigma, 1:1000). GAPDH, $\beta$-actin and $\alpha$-tubulin were used as loading controls. After incubation with appropriate secondary antibodies, signals were visualized by enhanced chemiluminescence (ECL). Each experiment was performed in duplicates and repeated three times.

\section{Cell migration assay}

Migration assays were performed in Transwell membrane filter inserts in 24-well tissue culture plates (BD Labware, Bedford, MA) with the pore size in polycarbonate membranes of $8 \mu \mathrm{m}$. Cells infected with lenti-MS1022X-miR-126 and lenti-pGIPZ for three days were detached, washed twice in PBS, and then 


\section{Cellular Physiology and Biochemistry}

Cell Physiol Biochem 2014;33:97-106

DOI: $10.1159 / 000356653$

Published online: January 17, 2014

Gong et al.: miR-126 Inhibits HSCs Activation
(C) 2014 S. Karger AG, Basel

www.karger.com/cpb

resuspended in DMEM containing 1\% FBS. A $100 \mu \mathrm{l}$ cell suspension containing $4 \times 10^{4}$ cells was added to each insert. DMEM containing 10\% FBS was added to the lower wells in the 24-well cell culture plate as chemoattractants. After a migration for $20 \mathrm{~h}$, inserts were fixed by $4 \%$ paraformaldehydeat and stained by Hoechst33342 at room temperature for $5 \mathrm{~min}$. Cells that did not migrate through the filters were removed by cotton swabs. Then cells were visualized under a fluorescent microscope. All experiments were repeated in triplicate.

Enzyme-linked immunosorbent assay (ELISA)

ELISAs were performed using commercial kits which were used for quantitative determination of collagen types I (Sigma, St. Louis, MO, USA) in HSCs culture supernatant three days after lentiviral infection.

Luciferase Reporter Assay

CRK 3'UTR were cloned into the pGL3 vectors by using SacI and XbaI restriction sites. 293FT cells were cultured in DMEM medium with $10 \%$ FBS and plated in 24 -well plate at a density of $5 \times 10^{4}$ cells per well. For each well, transfection mixture contained $100 \mu \mathrm{l}$ of Opti-MEM medium, $300 \mathrm{ng}$ of pGL3 luciferase reported vector and $20 \mathrm{ng}$ of renilla luciferase vector with Lipofectamine 2000 transfection reagent (Invitrogen). Luciferase assays were performed by the Dual-Luciferase Reporter Assay System (Promega). Each experiment was performed in duplicates and repeated three times.

\section{Immunostaining}

For inmunostaining, cells were washed twice with PBS and fixed with $4 \%$ paraformaldehydeat at room temperature for $10 \mathrm{~min}$. Fixed cells were permeabilized with $0.2 \%$ Triton X-100 for 8 min. Cells were then blocked in 2\% BSA in PBS containing 10\% FBS for $1 \mathrm{~h}$ at room temperature. Cells were stained with primary antibody (diluted at $1: 300$ ) overnight at $4^{\circ} \mathrm{C}$, then washed three times with PBS. Secondary antibody was diluted at 1:1000 and cells were stained for $1 \mathrm{~h}$ in the dark at $37^{\circ} \mathrm{C}$. Cells were stained with anti-collagen typeI, anti- $\alpha$-SMA, anti-CRK anti-F-actin and counterstained with Hoechst33342. Finally, cells were examined under confocal fluorescence microscope to capture both phase and fluorescent images. All experiments were repeated in triplicate.

\section{Results}

The endogenous expression of miR-126 is significantly decreased during HSCs activation

Primary rat HSCs were successfully isolated with the procedure as we previously performed. On average $3 \times 10^{7}-10^{8} \mathrm{HSCs}$ were harvested from each rat. These cells were small and round and appeared strong refraction when freshly isolated (Fig. 1A-a). After cultured for 2 days, the cells adhered to the wall of culture dish with an ellipse shape (Fig. 1A-b), then exhibited narrow antenna at day 7 (Fig. 1A-c), and presented asteroid membranous processes and appeared spindle-like shape at day 14 (Fig. 1A-d). qRT-PCR demonstrated that the markers for activated HSCs, $\alpha$-SMA and collagen type I, were very low in HSCs cultured in vitro at day 2 , slightly increased at day 7 and greatly enhanced at day $14(\mathrm{p}<0.01)$ (Fig.1B). Thus, HSCs cultrued at day 2 were deemed as the quiescent, HSCs at day 7 as the partially activated and HSCs at day 14 as the fully activated. Our results showed that miR-126 was gradually and significantly decreased during HSCs activation ( $\mathrm{p}<0.01)$ (Fig. 1C). Besides spontaneous activation of HSCs cultured in vitro, HSCs was also be activated by TGF- $\beta$ ( $2 \mathrm{ng} /$ $\mathrm{ml}$ ) stimulation for $24 \mathrm{~h}$ with significantly increased $\alpha$-SMA and collagen type I expression (Fig. 2B) as other researches did[16]. We found that miR-126 expression was also obviously decreased during HSCs activation stimulated by TGF- $\beta$ (Fig. 2 C). These results suggested that the expression of miR-126 was really significantly decreased during HSCs activation as our previous miRNA microarray showed.

Overexpression of miR-126 inhibits HSCs migration but not affects its proliferation

Then we explored the biological function of miR-126 during HSCs activation. First we restored the expression of miR-126 in HSC-T6 by infection of lenti-miR-126. The lentivius infection efficiency of HSC-T6 was evaluated by determining the percentage of green 


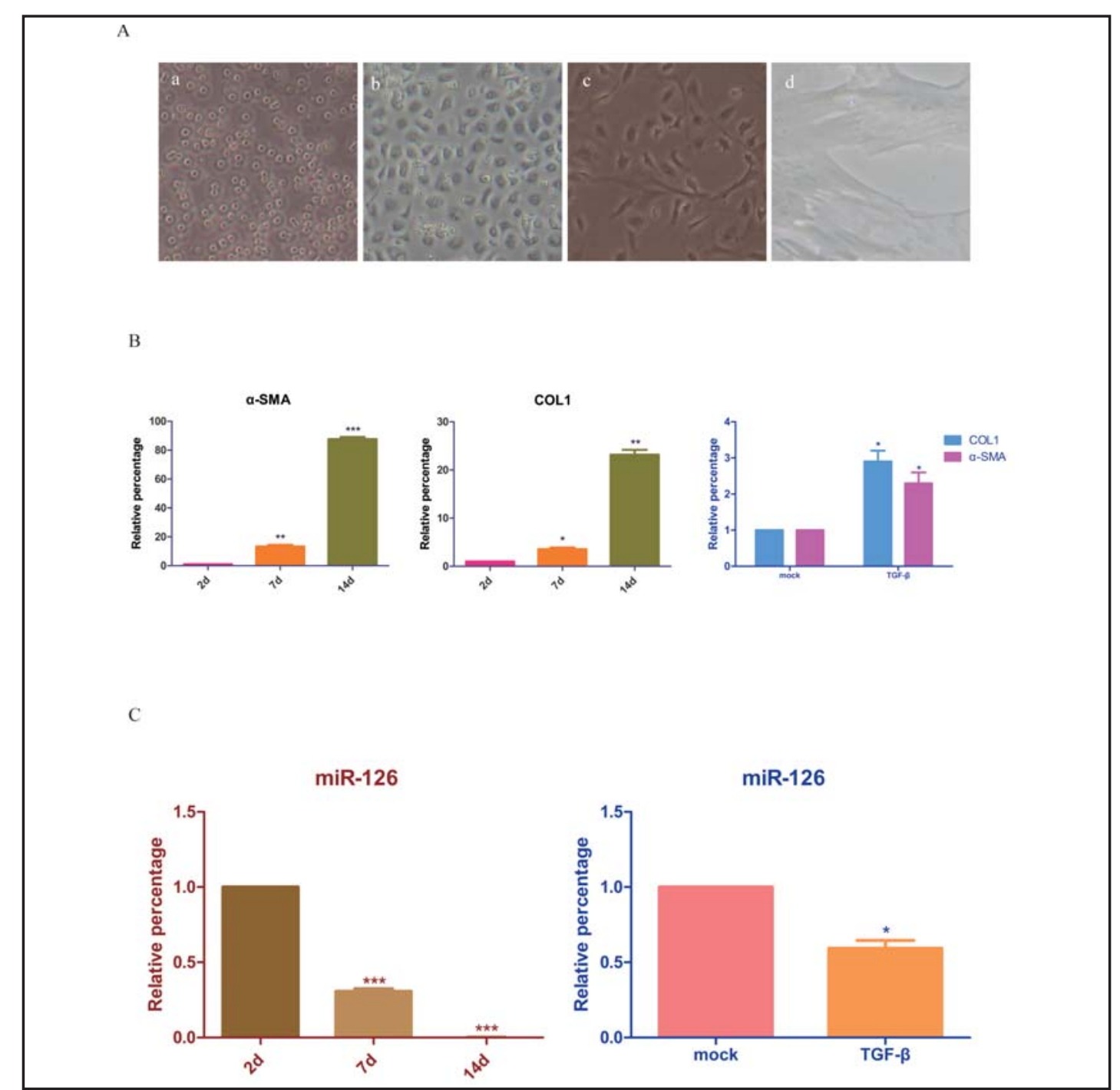

Fig. 1. The expression of miR-126 is significantly decreased during HSCs activation. (A) Cell morphology of freshly isolated primary HSCs(a), after cultured in vitro for 2 days (quiescent HSCs) (b), for 7 days (partially activated HSCs) (c) and for 14 days (fully activated HSCs). (B) The expression of $\alpha$-SMA and collagen type I was slightly increased at day 7 in partially activated HSCs and greatly enhanced at day 14 in fully activated HSCs. ${ }^{*} \mathrm{p}<0.05$ compared with day 2 in quiescent HSCs. The expression of $\alpha$-SMA and collagen type I were upregulated after TGF- $\beta$ stimulation. ${ }^{*} \mathrm{p}<0.05$ compared with control. The expression of $\alpha$-SMA and collagen type I were determined by qRT-PCR, normalized to $\beta$-actin. (C) miR-126 expression is gradually and significantly decreased during HSCs activation. ${ }^{*} \mathrm{p}<0.05$ compared with day 2 in quiescent HSCs. And miR-126 expression was also obviously decreased during HSCs activation stimulated by TGF- $\beta .{ }^{*} \mathrm{p}<0.05$ compared with control.

fluorescence protein (GFP)-positive cells. 80\% of HSC-T6 were successfully infected with lenti-miR-126 (MOI: 30) (Figs. 2A-a). And the expression of miR-126 was increased by 13-folds compared with the negative control measured by qRT-PCR (Figs. 2A-b). Then the role of miR-126 in regulating HSC-T6 proliferation and migration was studied. The results showed that there was no statistically change in proliferation in the miR-126-transfected HSCs assessed by CCK-8 (Fig. 2C). However, miR-126 restoration significantly inhibited HSC-T 6 migration by about $60 \%$ assessed by transwell assay compared with the negative control $(\mathrm{p}<0.01)$ (Fig. 2B). These results suggested that overexpression of miR-126 inhibited HSCs migration but not affected its proliferation. 
Fig. 2. Overexpression of miR-126 inhibits HSCs migration but not affects its proliferation. (A) Infection efficiency of HSC-T6 after 3 days. (a) Fluorescence microscope of HSC-T6 infected with fluorescently labeled miRNA after 3 days. The results showed that infection efficiency of HSCT6 was about $80 \%$ with MS1022X-miR-126 (MOI: 30). (b) The expression of miR-126 was increased by about 13-fold infected with MS1022X-miR-126 for 3 days in HSC-T6 assessed by qRT-PCR $\left({ }^{*} \mathrm{p}<0.05\right)$. (B) Upregulation of miR-126 inhibits HSC-T6 migration by about $60 \%$ assessed by transwell assay $\left({ }^{*} \mathrm{p}<0.05\right)$. (C) miR-126 did not affect HSC-T6 proliferation $(\mathrm{p}>0.05)$.

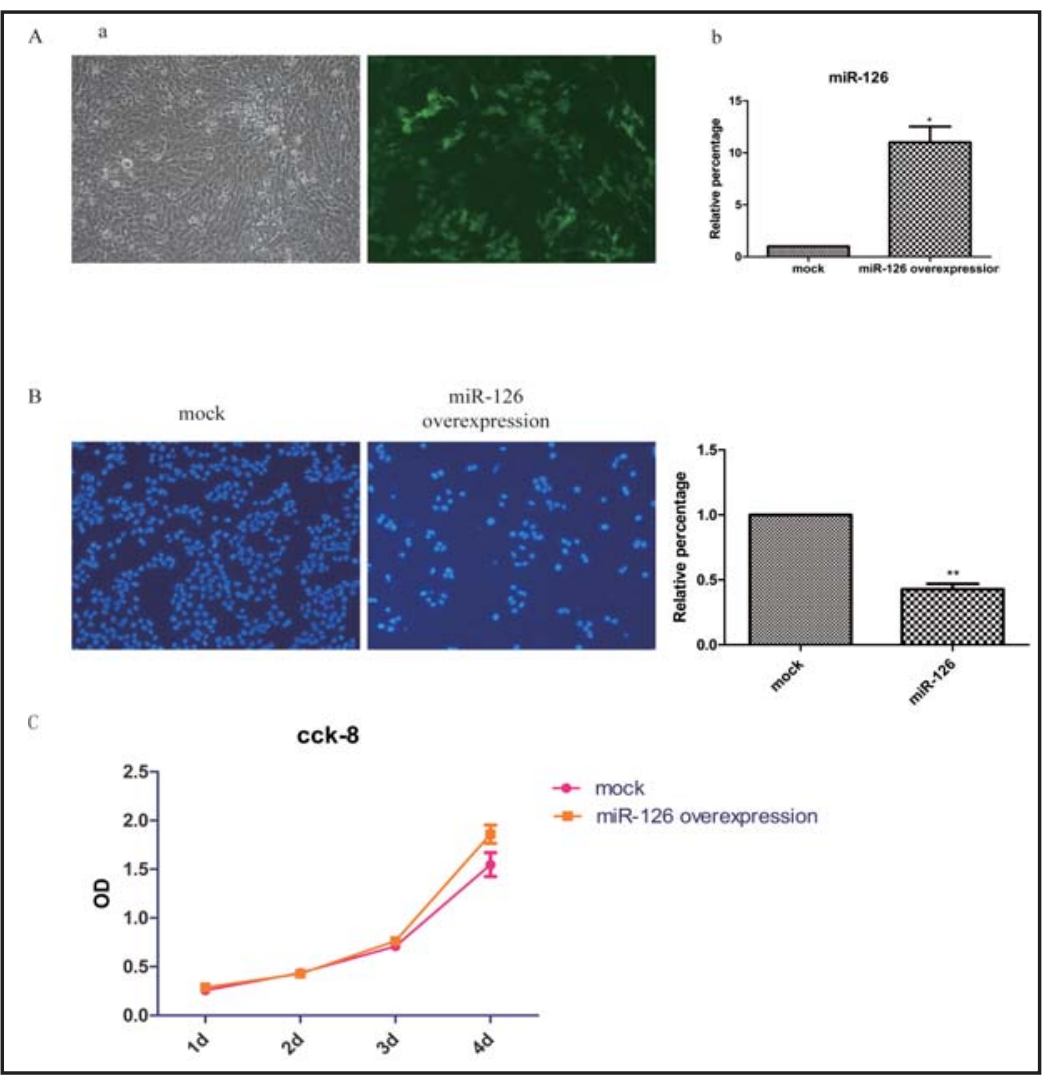

Overexpression of miR-126 ruduces $\alpha$-SMA and collagen type I expression in HSCs

Further, the effect of miR-126 on HSCs activation was also evaluated. First, the amount of collagen type I in HSCs culture supernatant three days after lentiviral infection was measured by ELISA. The results showed that collagen type I content in the media was decreased to $56 \%$ in miR-126 overexpression group compared with the negative control (NC) group (Fig. $3 A$ ). Then the expression of collagen type I and $\alpha$-SMA in HSCs three days after lentiviral infection were further measured by qRT-PCR, Western blot and immunofluorescence staining respectively. The results showed that the mRNA levels of $\alpha$-SMA and collagen type I were significantly descreased by about $70 \%$ and $50 \%$ after miR- 126 restoration compared with the negative control respectively $(\mathrm{p}<0.01)$ (Fig. 3B), and later Western blot and immunofluorescence staining results further confirmed miR-126 overexpression significantly reduced the protein levels of $\alpha$-SMA and collagen type I (Figs. 3C-E). These results demonstrated that overexpression of miR-126 significantly inhibits HSCs activation.

CRK is validated to be the target of miR-126 in HSC

CRK, which played an integral role in determining cell shape and locomotion, was predicted to be a target of miR-126 by bioinformatics approaches (Fig. 4A). First luciferase reporter assay was used to test whether CRK was a direct target of miR-126. The result showed that the 3'UTR of CRK could be directly targeted by miR-126 while the mutant 3'UTR of CRK was completely refractory to miR-126-mediated luciferase reporter repression (Fig. 4B). Then CRK expression either at mRNA or protein level in HSC-T6 were further measured by qRT-PCR, Western blot and immunofluorescence staining respectively. The results showed that CRK expression was gradually increased during HSCs activation $(\mathrm{P}<0.05)$ (Figs. 4D). Overexpression of miR-126 significantly decreased CRK expression at protein level while without any alteration in the mRNA level $(\mathrm{P}<0.05)$ (Figs. 4C and 4E). Besides, filamentousactin (F-actin), which played an integral role in determining cell shape and locomotion associated with CRK, was also obviously disturbed after miR-126 restoration showed by immunofluorescence staining (Figs. 4F). These findings indicated that CRK was a direct 


\section{Cellular Physiology \\ and Biochemistry}

Cell Physiol Biochem 2014;33:97-106

DOI: $10.1159 / 000356653$

Published online: January 17, 2014

(C) 2014 S. Karger AG, Basel

Gong et al.: miR-126 Inhibits HSCs Activation

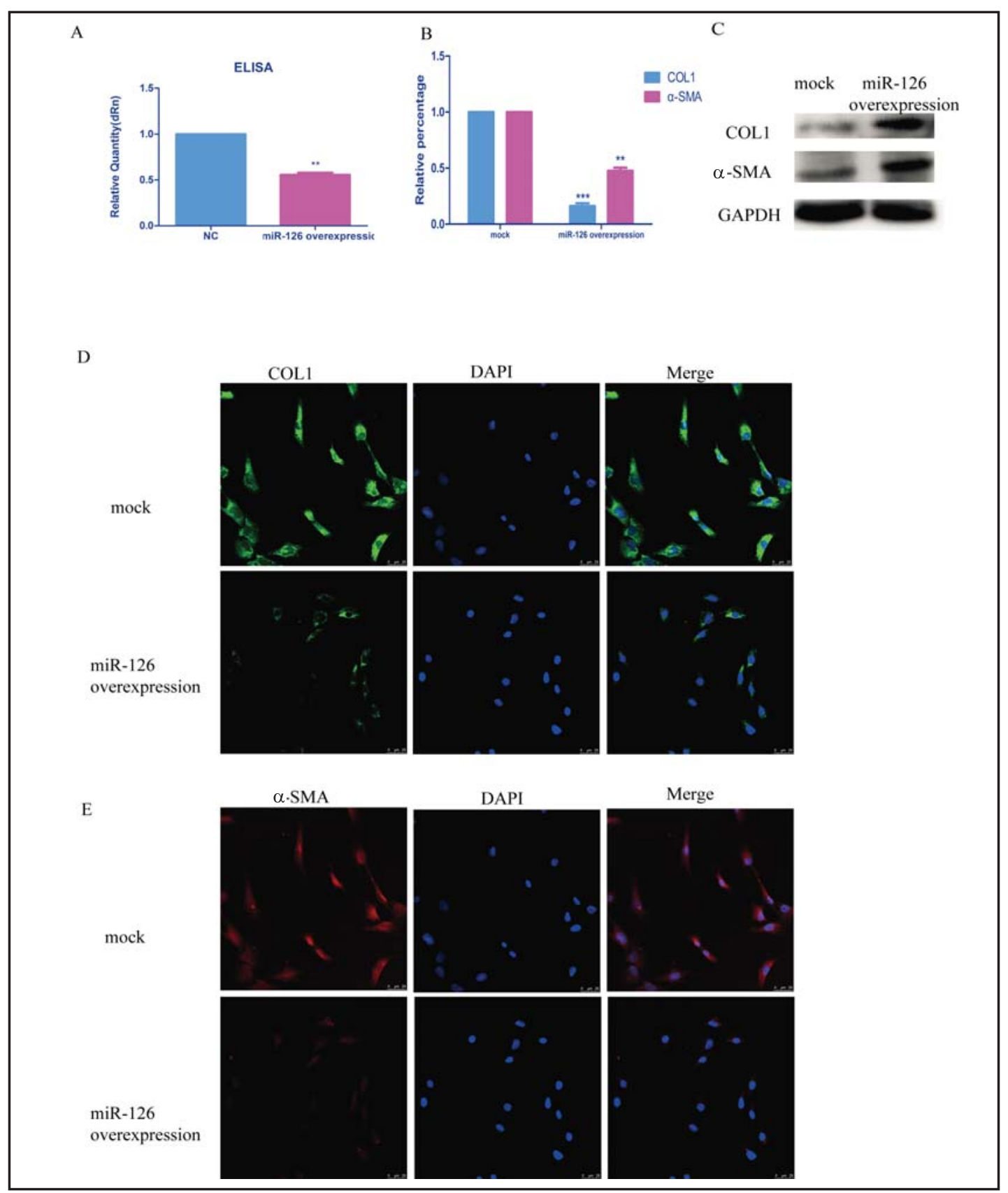

Fig. 3. Upregulation of miR-126 inhibits $\alpha$-SMA and collagen type I expression. (A) ELISA was used for quantitative determination of collagen types I content in HSC-T6 culture supernatant 3 days after lenti-virus infection. Collagen type I content in the media was decreased to $56 \%$ in miR-126 overexpression group compared with the negative control group. ${ }^{* *} \mathrm{p}<0.05$ compared with the negative control. (B) Overexpression of miR-126 decreased the mRNA levels of collagen type I and $\alpha$-SMA by about $70 \%$ and $50 \%$ respectively determined by qRT-PCR in HSCs $\left({ }^{* *} p<0.05\right)$. (C) The protein level of collagen type I and $\alpha$-SMA was decreased by overexpression of miR-126 assessed by Western blot. (D) Immunofluorescence staining of $\alpha$-SMA and collagen type I in HSCs after lenti-viral infection. Overexpression of miR-126 in HSC-T6 attenuated immunofluorescence staining of $\alpha$-SMA and collagen type I.

target of miR-126 and negatively regulated by miR-126 in HSCs. Overexpression of miR-126 inhibited HSCs activation and migration maybe through directly inhibiting CRK expression and indirectly disturbing F-actin. 


\section{Cellular Physiology $\quad$ Cell Physiol Biochem 2014;33:97-106

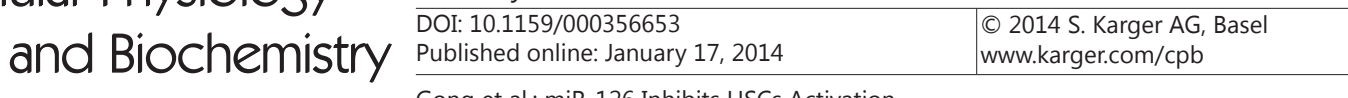 \\ Gong et al.: miR-126 Inhibits HSCs Activation}

Fig. 4. CRK is validated to be the target of miR-126 in HSCs and overexpression of miR-126 inhibits the expression of CRK. (A) The putative binding sequence of miR-126 in the CRK 3'UTR. Mutation was generated in the CRK3'UTR sequence in the complementary site for the seed region of miR-126. (B) Luciferase reporter assay was used to confirm the direct interaction between miR126 and target mRNA. The results showed that the 3'UTR of CRK could be directly targeted by miR-126 while the mutant 3'UTR of CRK was completely refractory to miR-126. (C) Overexpression of miR-126 in HSCT6 decreased the protein level of CRK determined by Western blot. (D) CRK was gradually and significantly increased during HSC activation. (E) Overexpression of miR-126 in HSC-T6 reduced immunofluorescence staining of CRK. (F) Immunofluorescence staining of $\mathrm{F}$ actin and CRK after lenti-

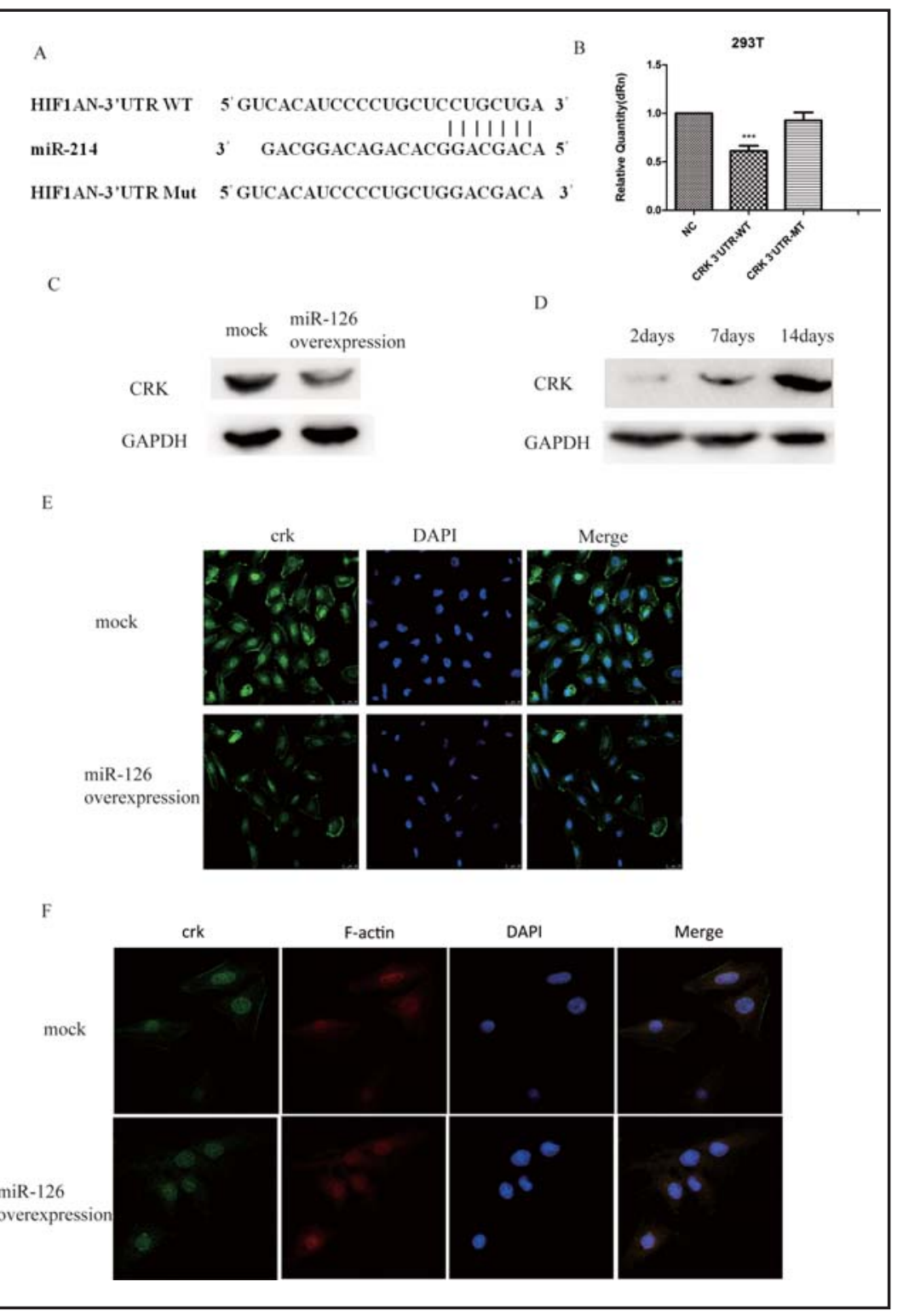

virus infection. Overexpression of miR-126 directly attenuated CRK expression and indirectly attenuated F-actin expression.

Overexpression of CRK promotes HSCs activation and decreases miR-126 expression

At last, the role of CRK played during HSCs activation was explored. First, CRK expression was obviously upregulated by 3.5-fold by qRT-PCR by infection of MS1022X-CRK into HSC-T6 compared with the negative control, which was further confirmed by Western blot result $(\mathrm{P}<0.05)$ (Figs. 5A and 5B). Then we found that CRK overexpression significantly increased $\alpha$-SMA and collagen type I expression and evidently decreased the miR-126 expression vice versa $(\mathrm{P}<0.05)$ (Figs. 5C and 5D). These results demonstrated that overexpression of CRK promoted HSCs activation and decreased miR-126 expression.

\section{Discussion}

Liver fibrosis is characterized by the excessive accumulation of extracellular matrix proteins and develops in most types of chronic liver disease, eventually leading to cirrhosis, 
Fig. 5. Overexpression of CRK promotes HSCs activation and decreases miR-126 expression. (A) The expression of CRK was increased by about 3.5 -fold 3 days after infection of lentipSB795-crk into HSCs assessed by qRT-PCR $\left({ }^{*} \mathrm{p}<0.05\right)$. (B) The protein level of exogenous CRK was upregulated by after infection of lenti-pSB795-crk into HSCs assessed by Western blot. (C) Overexpression of CRK in HSC-T6 increased the expression of collagen type I and $\alpha$-SMA. (D) Overexpression of CRK evidently decreased the miR-126 expression by about $70 \%$ assessed by qRT-PCR.

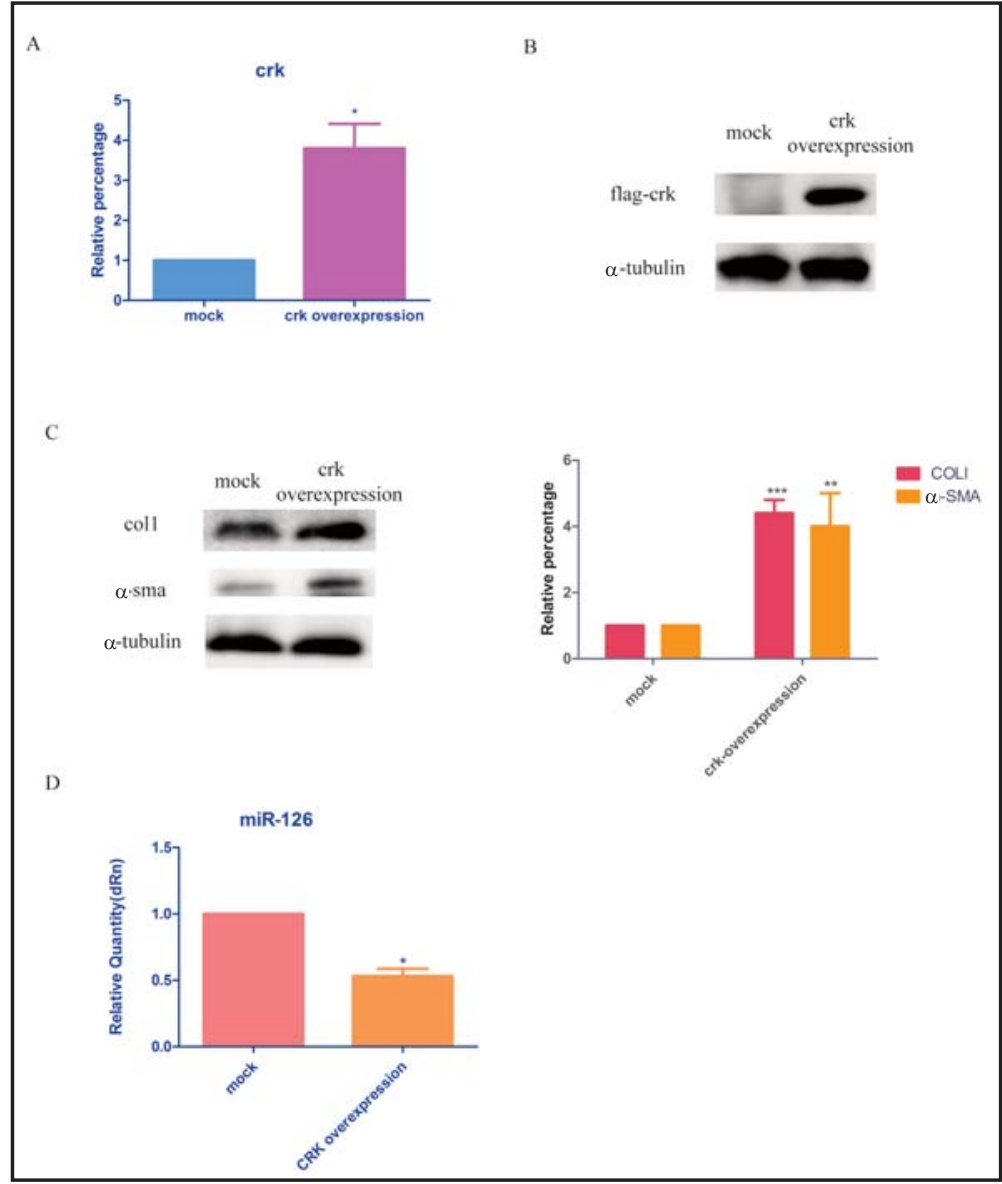

where activation of HSCs plays a crucial role in progression. Previous researches showed that miR-126 was obviously suppressed in many tumors including gastric cancer, breast cancer and lung cancer etc. These researches found that miR-126 usually inhibited the growth and invasiveness of cancers. Thus miR-126 was deemed as a tumor suppressor. In our previous study, the results of miRNA microarray showed miR-126 was significantly decreased during HSCs activation. Considering miR-126 often presented to be a growth suppressor and differentiation regulator in many cancer cells thus we hypothesize that miR-126 may also played essential roles in HSCs activation and differentiation. In this study, miR-126 was confirmed to be dramatically downregulated during HSCs activation and TGF- $\beta$ stimulation. Further the biological functions of miR-126 including cell proliferation, migration and differentiation during HSCs activation were determined. The results suggested that overexpression of miR-126 inhibited HSCs migration but not affects its proliferation. Besides, miR-126 restoration also significantly reduced $\alpha$-SMA and collagen type I expression thus inhibited HSCs activation. These results suggested that miR-126 played an essential part in HSCs activation. miR-126 might be a new negative marker and regulator of HSCs activation and liver fibrosis and it might be a promising therapeutic target of hepatic fibrosis.

In this study, CRK was found and validated to be a target of miR-126 in HSCs. CRK was a key signaling integrators of migration and invasion of cancer cells. CRK was often found to be significantly increased in many human cancers such as glioblastomas, lung cancer, breast cancer, synovial sarcoma, ovarian cancer and so on. And its expression level was often correlated with the invasiveness of tumors and aggressive and malignant behavior of cancer cells. CRK was able to enhance cell dispersal and invasion, and knockdown of CRK resulted in a significant decrease in migration and invasion of multiple malignant breast and other human cancer cell lines. Besides, Precious reports also demonstrated that CRK was required for formation of focal adhesions, and for PDGF-stimulated remodeling of the 


\section{Cellular Physiology and Biochemistry}

Cell Physiol Biochem 2014;33:97-106

DOI: $10.1159 / 000356653$

Published online: January 17, 2014

C) 2014 S. Karger AG, Basel

www.karger.com/cpb

actin cytoskeleton and cell migration [16]. CRK was predicted to be a target of miR-126 by bioinformatics approaches. Considering CRK played an integral role in cell migration, cytoskeletal regulation, morphology transition which were also closely correlated with HSCs activation, thus we hypothesized that CRK might also contribute greatly to HSCs activation. In this study, overexpression of miR-126 resulted in a significantly inhibition of CRK expression in HSCs and overexpression of CRK promoted HSCs activation in HSCs. Previous researches showed that CRK was associated with multiple signal transductions including PDGF signaling pathway, VEGF signaling pathway, MAP kinases ERK signaling pathway and so on, which also played important roles in HSCs activation [16-18]. However, the detailed mechanisms of HSCs activated by CRK was not clear yet and needed further explored. These results suggested that overexpression of miR-126 inhibited HSCs activation and migration might via inhibition of CRK expression.

\section{References}

1 Cheng K, Ye Z, Guntaka RV, Mahato RI: Biodistribution and hepatic uptake of triplex-forming oligonucleotides against type alpha1(I) collagen gene promoter in normal and fibrotic rats: Mol Pharm 2005;2:206-217.

-2 Kawada N: Evolution of hepatic fibrosis research. Hepatol Res 2011;41:199-208.

-3 Guo CJ, Pan Q, Li DG, Sun H, Liu BW: miR-15b and miR-16 are implicated in activation of the rat hepatic stellate cell: An essential role for apoptosis. J Hepatol 2009;50:766-778.

-4 Ji J, Zhang J, Huang G, Qian J, Wang X, Mei S: Over-expressed microRNA-27a and 27b influence fat accumulation and cell proliferation during rat hepatic stellate cell activation. FEBS Lett 2009;583:759-766.

-5 Venugopal SK, Jiang J, Kim TH, Li Y, Wang SS, Torok NJ, Wu J, Zern MA: Liver fibrosis causes downregulation of miRNA-150 and miRNA-194 in hepatic stellate cells, and their overexpression causes decreased stellate cell activation. Am J Physiol Gastrointest Liver Physiol 2010;298:G101-106.

6 Li J, Zhang Y, Kuruba R, Gao X, Gandhi CR, Xie W, Li S: Roles of microRNA-29a in the antifibrotic effect of farnesoid X receptor in hepatic stellate cells. Mol Pharmacol 2011;80:191-200.

7 Ogawa T, Enomoto M, Fujii H, Sekiya Y, Yoshizato K, Ikeda K, Kawada N: MicroRNA-221/222 upregulation indicates the activation of stellate cells and the progression of liver fibrosis. Gut 2012;61:1600-1609.

$>8$ Li WQ, Chen C, Xu MD, Guo J, Li YM, Xia QM, Liu HM, He J, Yu HY, Zhu L: The rno-miR-34 family is upregulated and targets ACSL1 in dimethylnitrosamine-induced hepatic fibrosis in rats. FEBS J 2011;278:1522-1532.

-9 Marquez RT, Bandyopadhyay S, Wendlandt EB, Keck K, Hoffer BA, Icardi MS, Christensen RN, Schmidt WN, McCaffrey AP: Correlation between microRNA expression levels and clinical parameters associated with chronic hepatitis C viral infection in humans. Lab Invest 2010;90:1727-1736.

- 10 Sekiya Y, Ogawa T, Iizuka M, Yoshizato K, Ikeda K, Kawada N: Down-regulation of cyclin E1 expression by microRNA-195 accounts for interferon-beta-induced inhibition of hepatic stellate cell proliferation. J Cell Physiol 2011;226:2535-2542.

11 Chen C, Wu CQ Zhang ZQ, Yao DK, Zhu L: Loss of expression of miR-335 is implicated in hepatic stellate cell migration and activation. Exp Cell Res 2011;317:1714-1725.

-12 Sun Y, Bai Y, Zhang F, Wang Y, Guo Y, Guo L: miR-126 inhibits non-small cell lung cancer cells proliferation by targeting EGFL7.Biochem Biophys Res Commun 2010;391:1483-1489.

13 Liu B, Peng XC, Zheng XL, Wang J, Qin YW: MiR-126 restoration down-regulate VEGF and inhibit the growth of lung cancer cell lines in vitro and in vivo. Lung Cancer 2009;66:169-175.

-14 Feng R, Chen X, Yu Y, Su L, Yu B, Li J, Cai Q, Yan M, Liu B, Zhu Z: miR-126 functions as a tumour suppressor in human gastric cancer. Cancer Lett 2010;298:50-63.

15 Guo C, Sah JF, Beard L, Willson JK, Markowitz SD, Guda K: The noncoding RNA, miR-126, suppresses the growth of neoplastic cells by targeting phosphatidylinositol 3-kinase signaling and is frequently lost in colon cancers. Genes Chromosomes Cancer 2008;47:939-946.

-16 Antoku S, Mayer BJ: Distinct roles for Crk adaptor isoforms in actin reorganization induced by extracellular signals. J Cell Sci 2009;122:4228-238.

17 Stoletov KV, Gong C, Terman BI: Nck and Crk mediate distinct VEGF-induced signaling pathways that serve overlapping functions in focal adhesion turnover and integrin activation. Exp Cell Res 2004;295:258-268.

- 18 Cho SY, Klemke RL: Extracellular-regulated kinase activation and CAS/Crk coupling regulate cell migration and suppress apoptosis during invasion of the extracellular matrix. J Cell Biol 2000;149:223-236. 